\begin{tabular}{|c|c|c|}
\hline Characteristics & $n=96$ & $\begin{array}{l}\text { Rate } \\
(\%)\end{array}$ \\
\hline Preoperative ureteral stent & 4 & 4.2 \\
\hline \multicolumn{3}{|l|}{ Long-term postoperative complications type ( $>30$ days) } \\
\hline - Urinary & 24 & 27.3 \\
\hline - Bowel/abdominal & 2 & 2.3 \\
\hline - Pulmonary & 0 & 0 \\
\hline - Infectious & 2 & 2.3 \\
\hline - Venous thromboembolism & 2 & 2.3 \\
\hline - Others & 21 & 23.9 \\
\hline \multicolumn{3}{|l|}{ Urological complications } \\
\hline - Infection & 16 & 18.2 \\
\hline - Ureteral obstruction/stenosis & 4 & 4.5 \\
\hline - Fistula (ureterovaginal/vesical) & 5 & 5.7 \\
\hline - Bladder injury & 1 & 1.1 \\
\hline $\begin{array}{l}\text { - Urological dysfunction (hypocontractility, incontinence, low- } \\
\text { compliance bladder) }\end{array}$ & 5 & 5.7 \\
\hline - Urinary sepsis & 2 & 2.3 \\
\hline - Haematuria & 0 & 0 \\
\hline - Acute urinary retention & 0 & 0 \\
\hline CT-urography performed & 14 & 15.9 \\
\hline \multicolumn{3}{|l|}{ Urological complications treatment } \\
\hline \multicolumn{3}{|l|}{ - Ureteral stent } \\
\hline o Unilateral & 5 & 5.7 \\
\hline o Bilateral & 5 & 5.7 \\
\hline - Suprapubic catheter & 2 & 2.3 \\
\hline \multicolumn{3}{|l|}{ - Nephrostomy } \\
\hline o Unilateral & 1 & 1.1 \\
\hline o Bilateral & 0 & 0 \\
\hline - Re-surgery & 5 & 5.7 \\
\hline o Fistula repair & 2 & 2.3 \\
\hline o Ureteral reimplantation & 2 & 2.3 \\
\hline o Fistula repair + Ureteral reimplantation & 1 & 1.1 \\
\hline o Nephrectomy & 1 & 1.1 \\
\hline
\end{tabular}

Abstract 871 Table 3 Oncological outcome

\begin{tabular}{lll}
\hline Characteristics & $\mathrm{n}=96$ & $\begin{array}{l}\text { Rate } \\
(\%)\end{array}$ \\
\hline Adjuvant treatment & 55 & 62.5 \\
- No & 17 & 19.3 \\
- RT & 17 & 19.3 \\
- RT/CT & 8 & 9.1 \\
Recurrence & & \\
Recurrence location & 2 & 2.1 \\
- Local & 1 & 1 \\
- Regional & 1 & 1 \\
- Locoregional & 1 & 1 \\
- PALN & 3 & 3.1 \\
- Distant and & & \\
multisite & 5 & 5.2 \\
Death of disease & $82.1 \%$ & \\
5-years DFS & $93.4 \%$ & \\
5-years OS &
\end{tabular}

CT=chemotherapy; DFS=disease free survival; OS=overall survival; PALN= para-aortic lymph nodes; $\mathrm{RT}=$ radiotheraoy. cervical cancer, patients with positive sentinel lymph node (SLN) in the intraoperative study and those with persistent or recurrent disease were excluded.

Result(s)* A total of 96 patients underwent surgery for earlystage cervical cancer. The pre-surgical stage was: IA $8.3 \%$, IB1 74\%, IB2 7.3\%, IIA1 9.4\%, IIB 1\%. Radical surgery was performed in 89 patients, fertility preserving surgery in 6 patients and radical colpectomy in 1 patient. Surgery approach was laparotomy, laparoscopy and vaginally in $15.6 \%, 78.1 \%$ and $6.3 \%$ of women, respectively. SLN biopsy was performed in $69.8 \%$ of patients. The mean operating time was 248.3 minutes and the mean hospital stay was 3.4 days.

The intraoperative and immediate postoperative complication rate $(<30$ days $)$ was $12.5 \%$ and $27 \%$ respectively. The Clavien-Dindo classification: II (61.5\%), III (38.5\%), IV and V $(0 \%)$. No statistical differences were found according to surgical approach and type of surgery in intraoperative $(p=1.00$; $\mathrm{p}=0.47)$ nor short-term complications $(\mathrm{p}=1.00 ; \mathrm{p}=0.5)$. Longterm complication rate (>30days) was $10.4 \%$. No differences were found according to surgical approach and type of surgery $(\mathrm{p}=0.28 ; \mathrm{p}=0.5)$. Urological complications occurred in 20 patients (20.8\%). Nine of them required ureteral catheter placement, 5 patients required re-intervention and 1 patient a nephrostomy.

Nearly $40 \%$ of the patients received adjuvant treatment: $58 \%$ only radiotherapy and $42 \%$ radiotherapy + chemotherapy. Six patients (6.25\%) presented urinary complications after $\mathrm{RT}$ and four patients $(4.2 \%)$ presented long-term urinary complications. No statistical differences in urological complications were found according to RT treatment $(\mathrm{p}=0.64)$.

The mean follow-up time was 39.7 months. Eleven patients $(11.5 \%)$ presented recurrence of their tumour process. Disease-free survival and overall survival at 5 years were $82.1 \%$ and $93.4 \%$ respectively

Conclusion* Surgery for early cervical cancer leads to urological complications in a considerable percentage of patients, however most of which are mild and self-limiting with medical treatment. Only a small group of patients will experience serious complications in the medium to long term.

\section{ADVANCED GYNECOLOGICAL CANCER: QUALITY OF LIFE ONE YEAR AFTER DIAGNOSIS}

B Jónsdóttir* , A Wikman, I Sundström Poromaa, K Stålberg. Uppsala University, Women's and Children's Health

\subsection{6/ijgc-2021-ESG0.580}

Introduction/Background* Gynecological cancer treatment can include various types of surgery, radiation and/or chemotherapy. It can leave irreversible changes on women's physical health and influence their psychological wellbeing affecting their quality of life. The aim of this study is to examine the quality of life of women with advanced gynecological cancer one year after diagnosis and identify possible predictive factors.

Methodology Women with endometrial, ovarian or cervical cancer included in the U-CAN (Uppsala-Umeå Comprehensive Cancer Consortium) database in years 2012-2019 were applicable for the study. Data from a general health questionnaire and the 36-item Short Form Survey (SF-36) were gathered along with information from medical records on histology, 
FIGO stage, type of treatment and surgical data. Inclusions criteria were completing the SF-36 at diagnosis and at one year follow up. Women with FIGO stage $\geq$ II wore considered to have advanced gynaecological cancer.

Result(s)* The final study population $(\mathrm{n}=372)$ included 150 $(40.3 \%)$ women in FIGO stage II or more. At diagnosis, their physical functioning and role functioning/physical scores were lower $(p=0.000, p=0.001)$ than for women with FIGO stage 1 . One year after diagnosis no difference was found in physical functioning of the two groups. Predictive factors for physical and mental health domains were identified by linear regression analyses; BMI, history of psychiatric illness and number of comorbidities were predictive for bot while education, smoking and FIGO stage $\geq$ II were not predictive.

Conclusion* Women with advanced gynecological cancer have a good quality of life one year after diagnosis compared to women FIGO stage I disease. BMI, history of psychiatric illness and number of comorbidities are predictive factors for physical and mental health.

\section{IMPACT OF SYSTEMATIC PELVIC LYMPHADENECTOMY ON SHORT TERM POSTOPERATIVE QUALITY OF LIFE IN PATIENTS WITH EARLY STAGE ENDOMETRIAL CANCER}

M Tanturovski*, I Aluloski, S Stojchevski, V Jovanovska, M Stojovski. University Clinic of Gynecology and Obstetrics, Department of Gynecologic Oncology, Skopje, Macedonia

\subsection{6/ijgc-2021-ESG0.581}

Introduction/Background* The objective of the study was to determine the potential impact of systematic lymphadenectomy vs. no lymphadenectomy on the perioperative change in QoL in patients undergoing surgical treatment for early stage endometrial cancer.

Methodology Patients scheduled for surgical treatment of clinically early stage endometrial cancer at the Department of gynecological oncology at the University Clinic of Gynecology and Obstetrics in Skopje, in the period January - December 2018 were approached for participation. Eligible subjects were divided into two groups: Group 1 (no LND) consisted of 60 patients who had hysterectomy plus bilateral salpingo-oophorectomy without lymph node dissection (LND); Group 2 consisted of 24 patients who had hysterectomy plus salpingooophorectomy plus systematic pelvic LND. Quality of life was quantified using a standardized and validated questionnaire (FACT-G) preoperatively and 30 days after surgical treatment. Result(s)* A total of 91 patients were recruited in the study. Of those, 7 patients (7.7\%) were excluded from statistical analysis: $6(6.6 \%)$ patients with incomplete questionnaires and one patient $(1 \%)$ who was unavailable for evaluation one month after surgery. The remaining 84 patients (92.3\%) were selected for analysis, 60 patients in group 1 (no LND) and 24 patients in group 2 (LND). The patients in the LND group exhibited statistically significantly lower postoperative scores for FACT-G (87.7 vs 75.8 for the no LND and LND groups respectively, $\mathrm{p}=0.002$ ), as well as for the physical wellbeing domain (23.4 vs. 20, $\mathrm{p}=0.004)$ and emotional wellbeing domain (20.7 vs 17, p=0.008). Twelve patients from the group with no lymphadenectomy (20\%) experienced a clinically significant decline in the postoperative QoL, compared to 12 patients $(50 \%)$ in the lymphadenectomy group $(p=0.006)$.
Conclusion* There was a significant decrease in the postoperative QoL 30 days after surgery in patients that undergo systematic pelvic lymphadenectomy for early stage endometrial cancer compared to patients that do not. Women undergoing surgery for endometrial cancer should be counseled about the potential benefits of surgical staging including LND, which can influence their postoperative treatment in a significant manner, as well as the possible negative impact of the treatment on the short-term QoL.

\section{HEALTH-RELATED QUALITY OF LIFE AFTER ROBOTIC SURGERY FOR ENDOMETRIAL CANCER - A PROSPECTIVE LONGITUDINAL FOLLOW-UP}

${ }^{1}$ A Lindfors*, ${ }^{2} \mathrm{P}$ Dahm-Kähler, ${ }^{3} \mathrm{~S}$ Järvholm. ${ }^{1} 1$ Department of Obstetrics and Gynecology, Institute of Clinical Science, Sahlgrenska Academy, University of Gothenburg, Gotheburg, Sweden; ${ }^{2} 1 D e p a r t m e n t$ of Obstetrics and Gynecology, Institute of Clinical Science, Sahlgrenska Academy, University of Gothenburg, Gothenburg, Sweden; ${ }^{3} 1$ Department of Obstetrics and Gynecology, Institute of Clinical Science, Sahlgrenska Academy, University of Gothenburg, Gothenburg, Sweden

\subsection{6/ijgc-2021-ESG0.582}

Introduction/Background* Health-related quality of life (HRQoL) are increasingly recognized as important parts of treatment evaluation. Still little is known about patients HRQoL after surgery and about their experiencing of symptoms. Endometrial cancer (EC) being a diagnosis with favorable prognosis and hence a large proportion of long-term survivors, the aspect of HRQoL becomes increasingly important. The study aims to explore how patients treated for EC with robotic surgery are affected in terms of health-related quality of life (HRQoL) and symptoms of anxiety and depression in the postoperative period. Furthermore, it aims to assess associations between HRQoL and patient characteristics.

Methodology Women scheduled for primary robotic surgery for EC during 1 year at a tertiary setting were invited to participate in the study. Factors including socioeconomic and civil status were obtained at baseline. Patient-reported outcomes were measured longitudinally by using the European Organization for Research and Treatment of Cancers (EORTC) Quality of Life Questionnaire Core 30 (QLQ-C30), its module for EC (EN24), the Generalized Anxiety Disorder Scale (GAD-7), and the nine-item Patient Health Questionnaire Depression Scale (PHQ-9) and followed prospectively from baseline to 2 weeks and 3 months postoperatively.

Result(s)* Sixty-four patients were included in the study. A significant decrease in Global health status (GHS) was seen at 2 weeks (from 69.8 to $62.7 ; \mathrm{p}=0.048$ ), with return to baseline levels at 3 months $(68.5 ; \mathrm{p}=0.32)$. Being unemployed $(\beta=-$ $36.35)$, having low income $(\beta=-12.78)$, and receiving adjuvant therapy $(\beta=-16.12)$ correlated with lower GHS at 3 months. The number of patients scoring above the clinical threshold for anxiety decreased from $17(27.0 \%)$ at baseline to $4(7.0 \%)$ at 2 weeks. Depressive symptoms were reported in $20 \%$ of patients at baseline and did not change significantly after 3 months.

Conclusion* Three months after robotic surgery for EC, there was no remaining negative impact on patients' HRQoL. However, receiving adjuvant therapy, low income, unemployment, or elevated depressive symptoms prior to EC diagnosis may motivate health care providers' increased attention. 